\title{
Relación entre Rasgos de Personalidad y Gestos: ¿expresamos lo que somos?
}

Relation between Traits of Personality and Gestures: do we express what we are?

Ángel Cuñado Yuste ${ }^{1}$

(1) Investigador independiente, Bilbao, España.

Email correspondencia: ayuste@euskalnet.net

\section{Resumen}

En este estudio se realiza una investigación en relación a la comunicación no verbal, en concreto al uso de los brazos y manos. Se muestra la relación existente entre los rasgos de personalidad definidos por el NEO PI-R y los gestos definidos por Paul Ekmany W allace Friesen como ilustradores, adaptadores, emblemas y reguladores emitidos en una situación de comunicación cara a cara o social. Los participantes son personas de ambos sexos y distribuidos al azar en parejas, con edades comprendidas entre 18 y 45 años. Los resultados del análisis indican que existe una significativa correlación entre las variables estudiadas: Neuroticismo con adaptador, apertura con ilustrador, amabilidad con ilustrador, amabilidad con reguladory responsabilidad con regulador (ver tabla 1). Estos datos refuerzan la hipótesis de que existe relación entre los gestos realizados con las manos y las características reflejadas en el NEO PI-R

Palabras Clave: Adaptadores, emblemas, ilustradores, reguladores, comunicación no verbal, pragmática.

\begin{abstract}
In this study an investigation is realized as regards the nonverbal communication, particularly in the use of the arms and hands. The existing relationship appears between the features of personality defined by the NEO PI-R and the gestures defined by Paul Ekman and Wallace Friesen such as illustrators, adapters, emblems and regulators expressed in a face to face or social communicative situation. The participants are persons of both sexes and distributed randomly in pairs, with ages between 18 and 45 years. The results of the analysis indicate that a significant correlation exists between the studied variables: Neuroticism with adapter, opening with illustrator, friendliness with illustrator, friendliness with regulator and conscientiousness with regulator (see table 1). These data reinforce the hypothesis of which relationship exists between the gestures carried out with the hands and the characteristics reflected in the NEO PI$R$
\end{abstract}

Keywords: Adapters, emblems, illustrators, regulators, nonverbal communication, pragmatics.

Fecha de envío: 19/04/2017

Fecha de aceptación: 25/07/2017 


\section{INTRODUCCIÓN}

Ya en 1979 Ray Birdwhistell propone que hace más de un siglo que los psiquiatras y los psicólogos han sido conscientes de que el comportamiento corporal y el gesto de cuerpo y manos son importantes fuentes de información en lo que respecta a la personalidad y a la sintomatología de los trastornos emocionales.

En lo que a la personalidad se refiere, se hace eco del término en su uso extendido al que refiere Bermúdez et al. (2011), entendiéndose que la personalidad engloba todas aquellas características, atributos y procesos psicosociobiológicos, cuya interrelación e integración posibilita identificar a cada persona como individuo único y diferente de los demás. Así la personalidad como propuso Allport (1961) posee una organización dinámica e interna, que crea patrones característicos de pensamiento y sentimientos y que, a su vez, es considerada más o menos estable en el tiempo siendo consistente (intra-individuo) y diferenciada de las otras personas (inter-individuos) en situaciones comparables, (Child, 1968). De lo anterior se deduce que la personalidad posee características que son internas y guían el comportamiento y que en esta investigación se verán reflejadas en los gestos. Así desde la teoría de los rasgos de la personalidad, no se podrá prescindir de la definición aportada por Cattell (1950) que la define como "aquello que permite una predicción de lo que una persona va a hacer en una situación determinada" (p.2).

Cuando se estudia la personalidad el modelo más extendido en la comunidad científica se establece en torno a los Cinco Grandes de personalidad, o Big-Five, un modelo de personalidad planteado en la década de los 60 que investigaciones posteriores han confirmado fehacientemente (Digman, 1990; Goldberg, 1993).

Se reproducen esquemáticamente los cinco factores principales del modelo del Big-Five que son evaluados mediante el NEO PI-R (Inventario de Personalidad Neo Revisado) de Costa y McCrae, (1999): Neuroticismo, implica una baja tolerancia para el estrés tanto de carácter físico (p. ej. el dolor) como psicológico (p. ej. la frustración). Extraversión, se relaciona con la tendencia de las personas hacia distintos tipos de actividades e intereses. Las personas extrovertidas se caracterizan por la sociabilidad, la necesidad de excitación y cambio, actuar en el momento, y son por lo general impulsivas. Suelen, además, ser optimistas y amantes del buen humor. También pueden ser agresivos, en el sentido de tener poca tolerancia a las frustraciones y poco control de sus sentimientos. Apertura a la experiencia, se refiere a la receptividad a nuevas ideas y experiencias, por lo que las personas bajas en este rasgo prefieren lo familiar, práctico y rutinario, mientras que las que puntúan alto en este rasgo están abiertos a nuevas experiencias, son curiosas e imaginativas. Amabilidad, significa el grado en que las personas son confiadas, generosas y se preocupan por los demás; aquellos con poca amabilidad son desconfiados, vengativos, obstinados e intolerantes. Y Responsabilidad, hace referencia al grado de organización, persistencia y motivación en tareas dirigidas a conseguir un objetivo, de modo que individuos altamente responsables o concienzudos son ambiciosos, trabajadores, competentes y organizados, y los de baja conciencia son perezosos, desordenados, indisciplinados y sin objetivos.

En relación a los gestos hace mucho tiempo que, Sir Francis Bacon (1891) puso la relación de gesto y lenguaje en forma de una simple analogía: "Como la lengua habla al oído, así el gesto habla al ojo" (p. 131).

Como refieren Krauss et al, en 1995, la investigación del Comportamiento No Verbal (CNV) en un amplio sentido se ha puesto cada vez más de moda, posiblemente por los avances conceptuales (véase, Ekman y Friesen, 1969; Mehrabian, 1972) y/o por mejoras en la capacidad de medida y análisis de estos comportamientos (p. ej. desarrollo de software de análisis y de los medios para el visionado de las grabaciones en alta definición). Debido a que el comportamiento no verbal es una parte implícita de la comunicación diádica, surge la intrigante pregunta de hasta qué punto estas conductas pueden ser indicativas de las características personales y tendencias conductuales de un individuo. En este punto es donde se desarrolla esta investigación.

Como refieren López, Gordillo y Grau (2016), hay una variedad de canales expresivos en relación a la investigación científica y dentro del Comportamiento No Verbal (ejemplo: facial, corporal, proxémico, prosódico emocional, háptico, apariencia, oculésica y cronémica).

Se puede observar que a lo largo de los años se han realizado diferentes investigaciones en los diferentes canales expresivos, dando mayor visibilidad a unos que a otros. A modo de ejemplo se puede ver, Murray (1971) quien ha presentado una fuerte evidencia de la relación entre la productividad verbal y la ansiedad; Markel y sus colegas 
(1964) probaron la hipótesis de que las impresiones específicas están determinadas por las cualidades de la voz. Las impresiones específicas se definieron como puntuaciones de los factores de Potencia y Actividad del diferencial semántico; Así mismo Markel (1969) también presentó una relación entre los perfiles de la calidad vocal y los perfiles MMPI en pacientes psiquiátricos. Cuñado (2010) hace referencia a la importancia de la proxémica en las relaciones terapéuticas. Klaus (1978) investigó el proceso de inferencia de la personalidad a partir de la calidad de voz. Krauss y otros (1995) hablan del valor comunicativo de los gestos de las manos en la conversación libre y la entrevista. Así mismo Freedman y colaboradores (1972) han observado una relación entre los movimientos de las manos y la diferenciación cognitiva representada por la dependencia de campo. Esta investigación ha sido realizada también por Witkin y Goodenough, (1977). El comportamiento visual también se ha relacionado con características de personalidad tales como la necesidad de afiliación (Exline, 1963), la deseabilidad social (Efran, 1968; Efran \& Broughton, 1966); Dominancia (Argyle \& Williams, 1969); Extraversión (Argyle \& Ingham, 1972, Argyle \& Williams, 1969, Kendon \& Cook, 1969, Mobbs, 1968, Rutter, Morley \& Graham, 1972). En 1980 Wiens, Harper, \& Matarazzo hacen referencia a la relación entre elementos de la personalidad como extroversión y neuroticismo en relación con la frecuencia de emisión del adaptador del entrevistado, los gestos ilustradores así como con miradas al entrevistador durante las entrevistas. Berry y Hansen (2000) analizan las relaciones diádicas en base a la extraversión y la aceptación percibida por los observadores. Levesque y Kenny (1993) realizaron un trabajo basado en las relaciones sociales, valorando el concepto de extraversión en función del comportamiento observado. Finalmente Kopple (2014) muestra diferencias individuales en frecuencia y tipo de producción de gestos en relación con características personales.

Aún así de estas investigaciones presentadas en la actualidad, la comunidad científica reconoce ampliamente que existe un vacío en la investigación del Comportamiento No Verbal, y hasta no hace mucho en la sociedad española el vacío era mayor.

Para apoyar la necesidad de investigación en este área y en nuestro país, así como para avanzar en la línea que ofrece la teoría de la comunicación en su aspecto pragmático, ampliamente desarrollada en otros países, véase Watzlawick
(1985), es necesario o incluso imprescindible que exista una serie de investigadores e investigaciones realizadas en y para nuestra cultura.

Fuera de nuestras fronteras se han realizado estudios por Ekman y Friesen (1969), y en nuestro país por López y colaboradores (2016) en cuanto a la expresión de las diferentes emociones representadas por microexpresiones de la cara. Por todo ello, un aspecto que parece interesante desarrollar es comprobar si existen relaciones entre los diferentes factores de personalidad y sus correlatos gestuales, mostrados mediante emblemas, ilustradores, demostradores de afecto, reguladores y adaptadores, en concreto expresados mediante los brazos y manos.

De las cinco categorías principales de comportamiento implícito o grupos de gestos propuestos por Ekman y Friesen, se utilizan únicamente tres en este experimento y se proponen investigaciones futuras en las restantes por tener, bajo el punto de vista de los investigadores, un tratamiento diferente.

En relación al objeto de estudio, se ha decidido prestar una mayor atención a los gestos realizados con movimientos de las manos y brazos, viendo la escasa investigación que existe, y por entender que supone una parte muy importante de la comunicación.

La hipótesis de trabajo que se valora es si existe algún tipo de relación entre los rasgos de personalidad reflejados en el NEO PI-R y los gestos realizados con las manos en una interacción cara a cara o social.

\section{MÉtodo}

\section{A. Sujetos/Participantes}

Los sujetos/participantes de la muestra son veinte personas $(\mathrm{N}=20)$ de las cuales el $15 \%$ son hombres y el $85 \%$ mujeres, con edades comprendidas entre los 18 y 53 años y con media de edad de 35 años y una desviación estándar de 11.3, todos ellos voluntarios (no pagados) para la realización "de un experimento sobre comunicación" que ejercen diferentes profesiones, y, en ocasiones, estudiantes de diferentes carreras.

\section{B. Instrumentos}

Se ha utilizado una Cámara Gessel con el sistema propio de grabación de vídeo y sonido; Así mismo se ha aplicado el test de personalidad NEO PI-R en formato de corrección 
automática; para el análisis de vídeos se ha usado el programa de análisis ELAN (EUDICO Anotador Lingüístico) desarrollado en el "Max Planck Institute for Psycholinguistics" en Nijmegen, Países Bajos, que es un instrumento de anotación que permite crear, corregir, visualizar y realizar búsquedas de anotaciones para datos de vídeo y de audio.

\section{Procedimiento}

A las personas participantes se les hace pasar por parejas, antes seleccionadas al azar, a la cámara Gessel y ante la videocámara que permanece detrás del espejo y ante el sistema de grabación de la propia cámara Gessel. Se realiza la grabación durante un período de 5 minutos al tiempo que se les dice "escogéis un sitio para sentaros y os sentáis a conversar"; el investigador, en los primeros momentos les informa de sus derechos en relación al experimento, solicitud de consentimiento informado, y se recoge la respuesta en vídeo.

Posteriormente se analizan y codifican los vídeos mediante el programa de análisis ELAN. Este programa permite la selección de las escenas con precisión desde el inicio de la acción o gesto hasta su final, con la posibilidad de anotar el significado del gesto ilustrador, adaptador, regulador u otro.

\section{Diseño}

La postura de esta investigación es planteada desde la Psicología correlacional, y utilizando un Paradigma correlacional respuesta-respuesta se trata de descubrir las posibles relaciones de covariación existentes entre múltiples variables.

Se utiliza una metodología observacional, con diseño descriptivo de tipo ex post facto o correlacional y cuantitativo por entender que es la que más se adecua al proyecto de investigación que se realiza; Se entiende que no se buscan relaciones de causalidad y que la forma de observar los datos que se obtenga, será ver la relación que existe o no entre las variables estudiadas. En esta investigación no se intenta encontrar relaciones entre lo verbal y no verbal o lo digital y analógico, esto es, entre el contenido y la relación en cuanto a congruencias o incongruencias entre ambos.

\section{E. Variables}

En este caso, las variables no son entendidas como dependientes o independientes, por ser de tipo correlacional y por no haber causalidad entre ellas. Estas variables son, por un lado, la variable características de personalidad (V1) con cinco factores principales (extraversión, neuroticismo, apertura, amabilidad y responsabilidad) reflejadas por la puntuación obtenida en un test de personalidad (NEO PIR) y, por otro lado, la variable total de gestos absolutos de las emisiones producidas (V2) por los participantes de los diferentes grupos de gestos, entendidos como ilustradores, adaptadores y reguladores. La amplitud y diferenciación de estos gestos son acotados por el inicio y la terminación del gesto, tanto en su forma activa como pasiva.

Es un análisis correlacional-descriptivo de las diferencias individuales existentes en las respuestas ante una situación dada y en contextos naturales, así como de las relaciones entre las respuestas observadas.

\section{REsultados}

Una vez obtenidos los resultados de la prueba test, así como los resultados en el análisis y codificación de los vídeos, y tras comprobar que no existe normalidad en algunos de los datos analizados se opta por realizar una prueba de correlación de rxy Spearman mediante el programa estadístico SPSS 15.0, porque permite comprobar las posibles relaciones de covariación existentes entre múltiples variables, aunque no su causación; las variables en este experimento son de tipo cuantitativo. Se trata de determinar si las dos variables están correlacionadas, es decir, si los valores de una variable tienden a ser más altos o más bajos para valores más altos o más bajos de la otra variable.

En la observación de los datos de la muestra trabajada, se ha encontrado que existe una correlación unilateral, positiva y significativa entre las variables Adaptador $y$ neuroticismo $\left(r=.481^{*}, p=.016\right)$, Ilustrador y apertura $\left(r=.472^{*}\right.$, $p=.018$ ), Ilustrador y Amabilidad ( $r=.517^{* *}, p=.010$ ), Regulador y amabilidad ( $r=.399^{*}, p=.041$ ), Regulador $y$ responsabilidad ( $r=.549 * *, p=.006$ ) al nivel de significación del *.05, y**.01 o lo que es lo mismo con una fiabilidad del $95 \%$ y $99 \%$ respectivamente (véase tabla 1 ).

También tras realizar un análisis de las facetas correspondientes a los factores principales se ha encontrado una correlación significativa entre: Neuroticismo - Adaptadory vulnerabilidad $(r=.438 *, p=.027$ ), ilustrador y vulnerabilidad ( $r=.450^{*}, p=.023$ ), Ilustrador $y$ 
$\operatorname{cordialidad}\left(r=.410^{*}, p=.036\right)$, Adaptador y Asertividad $(r=$. $\left.428^{*}, p=.030\right)$, Ilustrador $y$ asertividad $\left(r=.490^{*}, p=.014\right)$; Extraversión - Ilustrador y estética $\left.r=.494^{*}, p=.013\right)$; Apertura - Ilustrador y ideas $\left(r=.563^{* *}, p=.005\right)$, Ilustrador $y$ franqueza( $\left.r=.458^{*}, p=.021\right)$, Ilustrador y altruismo( $r=.574 * *$, $p=.004)$; Amabilidad-Ilustrador y sensibilidad a los demás ( $r=$ $\left..564^{* *}, p=.005\right)$, Ilustrador y competencia $\left(r=.509^{*}, p=.011\right)$, Regulador y competencia ( $\left.r=.457^{*}, p=.021\right)$, Regulador y sentido del deber $\left(r=.383^{*}, p=.048\right)$; Responsabilidad - Adaptador $y$ autodisciplina $\left(r=.-525^{* *}, p=.009\right)$, Regulador $y$ autodisciplina $\left(r=.504^{*}, p=.012\right)$, Adaptadory ansiedad ( $\left.r=.417^{*}, p=.034\right)$, Adaptador y depresión ( $r=.379 *, p=.050)$, Adaptador $y$ impulsividad ( $r=.440^{*}, p=.026$ ), al nivel de significación del *.05, y**.01.

Se observan correlaciones significativas (tabla1), se trata de correlaciones positivas pero no perfectas. Este conjunto de datos referente a los factores (figura 1), denominado diagrama de dispersión o nube de puntos, tiene interés como primera toma de contacto para conocer la naturaleza de la relación entre las variables que estamos trabajando. El grosor de la nube da una cierta idea de la magnitud de la correlación: cuanto más estrecha menor será el margen de variación en $\mathrm{Y}$ para los valores de $\mathrm{X}$ y, por tanto, más acertado los pronósticos, lo que implica una mayor correlación.

Figura 1 gráfico de dispersión X e Y

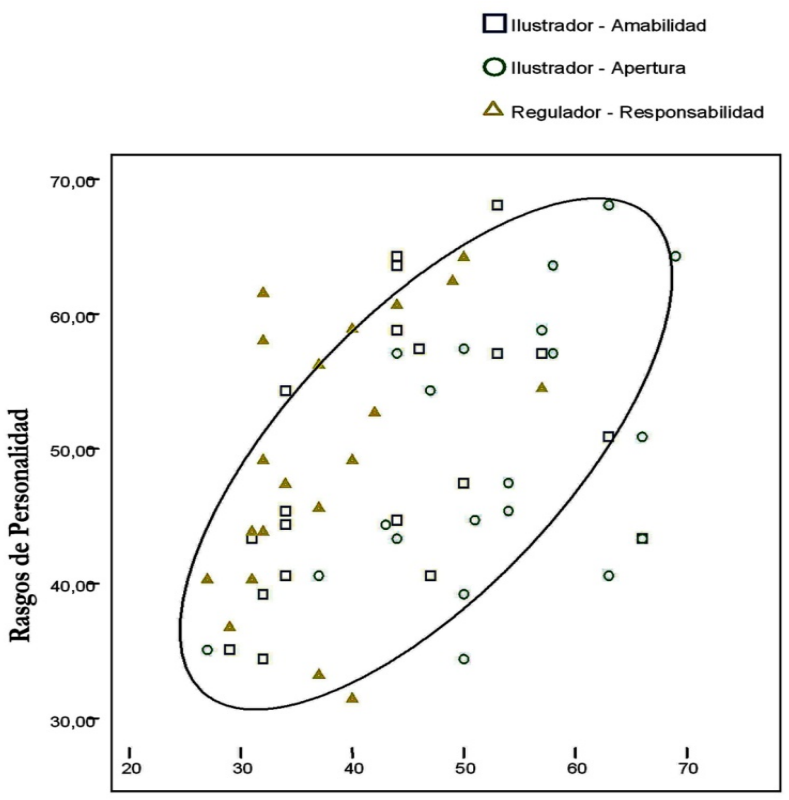

Gestos - Descriptores Conductuales
Tabla 1. Correlación entre gestos y factores de personalidad

\begin{tabular}{|c|c|c|c|c|}
\hline & & Adap. & Ilust. & Regul. \\
\hline \multirow[t]{3}{*}{ Neuroticismo } & $\begin{array}{l}\text { Correlación de } \\
\text { Pearson }\end{array}$ & ,481* &,- 156 &,- 200 \\
\hline & Sig. (bilateral) & ,016 & ,255 & 199 \\
\hline & $\mathrm{N}$ & 20 & 20 & 20 \\
\hline \multirow[t]{3}{*}{ Extraversión } & $\begin{array}{l}\text { Correlación de } \\
\text { Pearson }\end{array}$ &,- 224 &, 342 & ,130 \\
\hline & Sig. (bilateral) & ,172 &, 070 & ,292 \\
\hline & $\mathrm{N}$ & 20 & 20 & 20 \\
\hline \multirow[t]{3}{*}{ Apertura } & $\begin{array}{l}\text { Correlación de } \\
\text { Pearson }\end{array}$ & ,058 & ,472* & ,020 \\
\hline & Sig. (bilateral) & , 404 & ,018 & ,467 \\
\hline & $\mathrm{N}$ & 20 & 20 & 20 \\
\hline \multirow[t]{3}{*}{ Amabilidad } & $\begin{array}{l}\text { Correlación de } \\
\text { Pearson }\end{array}$ &,- 055 &, $517 * *$ & ,399* \\
\hline & Sig. (bilateral) &, 409 &, 010 &, 041 \\
\hline & $\mathrm{N}$ & 20 & 20 & 20 \\
\hline \multirow[t]{3}{*}{ Responsabilidad } & $\begin{array}{l}\text { Correlación de } \\
\text { Pearson }\end{array}$ &,- 308 &, 140 &, $549 * *$ \\
\hline & Sig. (bilateral) &, 093 & ,279 &, 006 \\
\hline & $\mathrm{N}$ & 20 & 20 & 20 \\
\hline
\end{tabular}

\section{DiscusióN}

Se ha realizado un análisis unilateral en espera de que la relación que se obtenga sea en una dirección determinada (ejemplo: que los más amables y con mayor apertura a la experiencia utilicen más ilustradores y no lo contrario).

Los datos obtenidos en experimentos como los realizados por Berry y Hansen (2000), los observados en los trabajos de Levesque y Kenny (1993) y otros reflejados anteriormente muestran algún tipo de relación entre los canales de la comunicación no verbal y la personalidad. Más concretamente Kopple (2014) obtiene resultados que apoyan la propuesta de relación entre personalidad y conducta no verbal a través de la emisión de gestos realizados con las manos.

Una de las limitaciones que se considera importante es que la muestra que se ha manejado es bastante pequeña $\mathrm{N}=20$, aún así los resultados son prometedores en cuanto a la realización de un estudio futuro más amplio y con mayor cantidad de participantes.

Se considera conveniente la realización de una replicación del trabajo experimental, eso sí con mayor cantidad de participantes y con la utilización de un acuerdo 
inter-jueces a la hora de establecer los gestos y tipos de gestos a utilizar. Pues se ha observado que en la literatura específica hay aún una gran diversidad en los criterios a la hora de decidir la nomenclatura referente a los tipos de gestos, no habiendo acuerdo en cómo referirse a los mismos.

\section{A. Conclusiones}

De los trabajos realizados hasta la fecha se intuye que

\section{REFERENCIAS}

Allport, G. W. (1937). Personality: a Psychological Interpretation: Oxford, England: Holt.

Allport, G. W. (1961). Pattern and Growth in Personality: Holt, Rinehart and Winston.

Allport, G. W. (1965). Psicología de la personalidad. Buenos Aires Paidós.

Argyle, M. (1978). Bodily Communication. London: Methuen \& Co Ltd. (Publicación original 1975). Reprinted 1976

Argyle, M., \& Ingham, R. (1972). Gaze, mutual gaze, and proximity. Semiotiea, 6, 32-49.

Argyle, M., \& Williams, M. (1969). Observer or observed? A reversible perspective in person percteption. Sociometry, SB, 369-412.

Bacon, F. (1891). The Advancement of Learning, Book (William Aldis Wright, ed.) Clarendon Press.

Berry, D. S., \& Hansen, J. S. (2000). Personality, nonverbal behavior, and interaction quality in female dyads. Personality and Social Psychology Bulletin, 26(3), 278-292.

Bermúdez, J., Pérez, A. M., Ruíz, J. A., Sanjuán, P., \& Rueda, B. (2011). Psicología de la personalidad. Madrid: UNED.

Birdwhistell, Ray L. (1979). Kinesics and Context: Essays on Body Motion Communication [El lenguaje de la expresión corporal] (Desmonts, Antonio J.,trad.) Barcelona: Editorial Gustavo Gili, S.A. (Publicación original 1970)

Cattell, R. B. (1950). Personality: A Systematic Theoretical and Factual Study. New York: McGraw Hill.

Costa, P. T., Jr., \& McCrae, R. R. (1999). Inventario de Personalidad NEO Revisado (NEO PI-R). Inventario NEO reducido de Cinco Factores (NEO-FFI). Manual. Madrid: TEA.

Cuñado Yuste, A. (2010). Comunicación no verbal y orientaciones de intervención en la proxémica de la familia en sesión terapéutica. Mosaico, Vol. 44. pp. 68-77

Child, I. L. (1968). Personality in culture. In W. W. L. E. Borgatta (ed.), Handbook of Personality Theory and Research (pp. 80- 101). Chicago: Rand McNally.

Digman, J. M. (1990). Personality Structure: Emergence of the FiveFactor Model. Annual Review of Psychology, 41, 417-440.

Efran, J. S. (1968). Looking for approval: effects on visual behavior of approbation from persons differing in importance. Journal of Personality and Social Psychology, 10(1), 21. existe relación entre la personalidad y la conducta no verbal. Pues teniendo en cuenta las limitaciones expuestas anteriormente, se puede concluir que se acepta la hipótesis de trabajo de que existe algún tipo de relación entre las variables rasgos de personalidad y el Comportamiento No Verbal expresado en el canal de gestos y en su variante movimientos realizados con las manos en una interacción cara a cara o social.

Efran, J. S., \& Broughton, A. (1966). Effect of expectancies for social approval on visual behavior. Journal of Personality and Social Psychology, 4(1), 103.

Ekman, P. \& Friesen, W.V. (1969). The Repertoire of Non Verbal Behavior. Semiotica, 1, pp.49-98.

Exline, R. V. (1963). Explorations in the process of person perception: Visual interaction in relation to competition, sex, and need for affiliation. Journal of personality, 31(1), 1-20.

Freedman, N., O'Hanlon, J., Oltman, P., \& Witkin, H. A. (1972). The imprint of psychological differentiation on kinetic behavior in varying communicative contexts. Journal of Abnormal Psychology, 79(3), 239.

Goldberg, L. R. (1993). The structure of phenotypic personality traits. American Psychologist, 48, 26-34.

Klaus, R. (1978). Personality inference from voice quality: The loud voice of extroversion. European Journal of Social Psychology, 8, 467-487.

Kendon, A., \& Cook, M. (1969). The consistency of gaze patterns in social interaction. British Journal of Psychology, 60(4), 481-494.

Kopple, K. (2014). Individual differences in frequency and type of gesture production: Relationship to personal characteristics (Doctoral dissertation, Bryn Mawr College).

Krauss, R. M., Dushay, R. A., Chen, Y., \& Rauscher, F. (1995). The communicative value of conversational hand gestures. Journal of Experimental Social Psychology, 31, 533-552.

Levesque, M. J., \& Kenny, D. A. (1993). Accuracy of behavioral predictions at zero acquaintance: A social relations analysis. Journal of Personality and Social Psychology, 65(6), 1178.

López Pérez M., F. Gordillo León \& M. Grau Olivares (Coords.) (2016). Comportamiento no verbal: más allá de la comunicación y el lenguaje. Madrid: Pirámide.

Markel, N. N., Meisels, M., \& Houck, J. E. (1964). Judging personality from voice quality. The Journal of Abnormal and Social Psychology, 69(4), 458.

Markel, N. N. (1969). Relationship between voice-quality profiles and MMPI profiles in psychiatric patients. Journal of abnormal psychology, 74(1), 61.

Mehrabian, A. (1972). Nonverbal Communication. In J.K. Cole (Ed.), Nebraska Symposium on Motivation, 1971. Lincoln, NE: University of Nebraska Press. Vol. 19, pp. 107-161. 
Cuñado, A. (2017). Relación entre Rasgos de Personalidad y Gestos: ¿expresamos lo que somos? Behavior and Law Journal, 3(1), 35-41.

Mobbs, N. A. (1968). Eye-contact in relation to Social Introversion/Extraversion. British Journal of Clinical Psychology, 7(4), 305-306.

Murray, D. C. (1971). Talk, silence, and anxiety. Psychological Bulletin, 75(4), 244-260.

Rutter, D. R., Morley, I. E., \& Graham, J. C. (1972). Visual interaction in a group of introverts and extraverts. European Journal of Social Psychology, 2(4), 371-384.

Watzlawick, P., Helmick Beavin, J. \& Jackson, Don D. (1985). Pragmatics of Human Communication [Teoría de la comunicación humana: Interacciones, patologías y paradojas] ( $4^{\text {a }}$ edición). Barcelona: Editorial Herder S. A. (Publicación original 1967)

Wiens, A. N., Harper, R. G. \& Matarazzo, J. D. (1980), Personality correlates of nonverbal interview behavior. Journal of Clinical Psychology, 36(1), 205-215.

Witkin, H. A., \& Goodenough, D. R. (1977). Field dependence and interpersonal behavior. Psychological Bulletin, 84(4), 661. 